\title{
Cross-platform Smartphone Emergency Reporting Application in Urban Areas using GIS Location based and Google Web Services
}

\author{
Swapnil R. Rajput, Mohd Sohel Deshmukh, Karbhari V. Kale, PhD \\ Department of Computer Science \& Information Technology, \\ Dr. Babasaheb Ambedkar Marathwada University, Aurangabad, (MS). India
}

\begin{abstract}
Smartphone's are existence utilize for various ranges of vigorous activities including social media, messaging, a chart, context directorate in addition for location applications. Emergency never comes with prior information. In real world case such emergencies and reporting them is a real challenge. This paper contains the existing systems detail survey and proposed system to overcome common problem of having manual intervention while reporting emergency. We are proposing the new idea for emergency detection and reporting. This system will record and report emergency in real time. Reporting of emergency comes with two ways either the person at emergency situation can report directly by Smartphone with nearest emergency facility or by reporting and response team comes with emergency facility like medical, fire, police. It works in three steps as detection of emergency with current location, user data collection and processing and reporting to web application. This is the clientserver GIS and Smartphone application for the person at emergency situation. the person at emergency select the emergency service such as in client application medical, fire, police, then it detect the user current location along with information name of the person and contact number(person's identity) for providing service then register, after registering their will be two options either the person at emergency select its own nearest service such as for medical nearest hospitals to provide medical facility, in case of fire nearest fire stations and police in criminal, illegal activity etc and second way after registering details information will placed onto server database so that rescue team will get information and provide appropriate services to the person. This proposed work, we will record all emergency details of rescued person with location onto maps, GIS will provide detection of emergency person coordinates (latitude and longitude) tracked by GPS and also provide plotting coordinates onto maps in the web application. Current project also Tracks location of person and sharing it with helping message to its family members, friends or any by selecting contact number from Smartphone and share via SMS or any social applications like face book, email, whatsapp so that front person understand person is in emergency and they will help them.
\end{abstract}

\section{Keywords}

Phonegap, Google Maps API, GIS, LBS, Google Maps, Emergency Reporting, GPS

\section{INTRODUCTION}

Today's world population more than half people's lives in urbanized areas and in most countries of the world the citizen uses Smartphone's. Smartphone's are becoming the first communication and computing media for daily tasks for the people. With uses of social media, "netizens" are now pleasant with very often updating their current going activities or social profiles with their locations [1]. This new reference of information, signals from social micro blog programme, has been found to be principally useful in relief operations and disaster management. For example, in July 2012, during the Beijing floods, people used twitter to provide information about the flooded areas and associatively developed a live crisis map of the floods impact using Google Maps.

According to a report from researchers and survey, Smartphone or mobile applications now account for $15 \%$ of all Internet traffic, with 1.5 billion customers globally. Whether you are a mortar and brick retailer or an ecommerce web site the need to go Smartphone is greater than everything. With Smartphone market share growing year over year, Smartphone applications are the new web site. If you don't have these available you are missing a major market. Smartphone adoption has accelerated like never before [2].

In today's smart world, Technology is increasing rapidly by human in everywhere and every time. Automatic systems value increased in their life for more comfortable. Smartphone's have various functions to support different user intersects.GPS in Smartphone's offer location geo-position and this will be helpful when someone in emergency situation. Consequently, in this research paper designed to be used by someone in emergency to assist emergency services like police, fire stations, ambulance for medical services.

As per Indian government's ministry of road transport and highways stations report through the calendar year 2010, there were near to 5 lakh road accidents happened in India, which effected in more than 1.3 lakh deaths with inflicted injuries on 5.2 lakh persons. These numbers changes into one road accident every minute and one road accident death every four minutes. Sadly more than half the injured are in the economically active age group of 25-65 years. The loss of main bread winner can be unsuccessful. (Source: GOI, Ministry of road transport, research wing and highways, New Delhi). The global accident statics indicate that the accidental rates per license vehicle are very high in developing countries. Even more road accidents have been exhibit to cost around $1 \%$ of annual gross national product (GNP) resources for the developing countries, which they can poorly-afford to lose[3].Hence there is a need to reduce this rate and provide some counter measures. It has been calculated that India presently accounts for nearly $10 \%$ of road accident fatalities globally. In inclusion, more than 1.3 million people are seriously injured on the Indian roads every year [4]. 
Presently in India, different emergency communication and response Systems are in place for police department, fire brigade, ambulance, disaster management, civil protection etc. These systems are accessed through different numbers such as 100(Police), 101(Fire brigade), 102(Ambulance), 108 (Disaster Management in case of emergency) [5].For example somewhere at city personal from command center and person who called for emergency, this system assist with response without any delay in service. System will act as interface between agencies and the one who alert an incident. Through the Response got from source geo-Location the response team gets the shortest distance to reach to the place of incident to avoid delay. Queries will be resolved based on the severity, urgency, need.

In all such situations person who is in emergency will not be in position to get services to inform disaster management team and this is the worst situation where needy needs help but able to seek it. So by any means if emergency is detected and reported to disaster management team, then this team will be in position to rescue person in emergency. Getting nearest places placed important factor in case of emergency to get facilities from service providers like medical, fire and police in case of disaster. Proposed client side will get all nearest service places with Geo-coded address, contact number so that rescue person contact directly and placed service. Sharing current location to your family or any friends also important to rescue from situations, current application provide facility to share GPS coordinated message with Google maps integration to any contact number from smart phone.

The satellite image of Aurangabad city is utilized to extract the field of interest in current research paper, but user can use this application at any city. As it uses Google places API as a spatial data so that mapped placed information extracted from it and used as for emergency purpose. For demonstration, we consider Aurangabad city as a study area to facilitate services to the emergency person. The main objective of this paper is to allocate emergency services for the rescue person, by using Smartphone technology time required to report an incident and to determine geo-location precisely. This will reduce time required for emergency personal to reach incident location. In this paper, section I focuses on basic needs of work done, Section II have related work of previous literature survey, section III specify the proposed system architecture, section IV focus on experimental work done with the technologies used and section $\mathrm{V}$ specify the conclusions and future work to be done.

\section{RELATED WORK}

Emergency never coordinate with prior information, the detailed information has to be filled to saves lives at incident places. Proposed Emergency reporting application is for Urban Area of Aurangabad city which is located with coordinate's $19.88^{\circ} \mathrm{N} 75.32^{\circ} \mathrm{E}$. Our proposed work is for Urban Areas at Aurangabad city as a case Study. Emergency Management Literature categories with two phases i.e. existing systems reviews \& current Different technologies for emergency management.

\subsection{Existing Systems}

For Emergency or Non-Emergency cases, Aurangabad Municipal Corporation (AMC) provided Online Complaint Web-System, where user can register their complaint with detailed information such as type of complaint, it's Subtype, ward wise Complaint registered, Landmark, Geo- Location and Description followed by Details of Complaint Registered
Person then After registering complaints Token will generated for Registered Complaints then onto respective department these requested complaints forwarded. User can confirm Status of the complaints online [6] [7].

Emergency service System 100 provided for calling police. Emergency incidents reported from 1-0-8 require basic information so that call taker dispatches appropriate services to the incident person.

\subsection{Current systems for emergency reporting}

\subsubsection{ELERTS App}

This is widely used mobile application in all over America. This system is centralized and available in both application and web format. Using this application the person on emergency site can take photo of it, which automatically uploaded to website and other application user gets the alert regarding such emergency along with location and photo. So being one 1st hand responder of any emergency, one can help other via this application [8]. ELERTS Features Include: Two-way communication: once user get alert message on his/her phone, they can also reply with any other detail information regarding that emergency. Here rescue teams or any other helper and suggest the directions to get out of such situation. Reports: every Smartphone user who has this application installed on their cell phone can send 1st hand report to others. Report may include text and pictures. This is actually triggering point of this application where user witness about emergency and send across. Photos: sharing the live images with security personal or any other nearby user is great feature supported by this application. GPS \& Mapping: using latest technologies like Google Maps and geo location parameters, application locates the emergency place and advice others on the issue with location details.

\subsubsection{HelpMe}

Another interesting mobile app which deals with disaster environment called HelpMe [9]. It introduces new approach of building the ad-hoc network using Wi-Fi to enable smart phones to communicate during disaster time. it works without help of any telephone providers network, and smartly forwards the message on hop-to-hop basis. It also make decides to forward the message by using routing algorithms. It has centralized HelpMe server to record the all happening about emergency, once service is restored. This information from cell phone intended to know the missing person.

\subsubsection{First Aid Application on Mobile Device}

FAD is expanded to give some directions for preliminary taking care of victim/patient from the Android Smartphone device. The navigation function using Google Maps API is executed in this paper for searching a suitable direction to the nearest search/nearest hospital. Therefore, in the case of emergency, this function will be activated and navigate victims/patients to the nearest hospital with the shortest path [10].

\subsubsection{GreatCall}

GreatCall [11] gives you easy-to-use and reliable/user friendly wireless services. This is wireless system very similar to normal mobile phone application but with one button device. Five stars (5 stars) is most used services in which person in emergency can talk with service representation directly upon pressing that help button. Quick response with trained agent on call is main feature of such services. This is mostly used as personal advisable device in US, as most of citizen are staying 
alone in home and getting noticed by someone in emergency is important during emergency.

\section{SYSTEM OVERVIEW}

The development of the client-server system is divided into the development of sub modules like application development , server application development and the environment used, technologies used, database server with platform to be used in all mobile operating systems.

\subsection{System Architecture}

The system architecture is shown in fig.1. The system is divided into 3 parts, the development of the client side which is cross- platform Smartphone app consist of different emergencies units and reporting system such as medical emergency for providing ambulance services, fire services and police services nearby location from the user current location. The Google web services used for getting the nearby search places from the current location of the user in Smartphone app which provide mapping of the nearby places on to the Google mobile maps along with fetching required information that user wants at the time of emergency such as geo-location of place, mapping facility to see on to the Google maps. In this approach we have used different technologies like JQuery, Phonegap (Apache Cordova), Html 5, JavaScript, CSS for the development of the client side emergency reporting App. Html5 provide great platform to developed cross- platform mobile applications by using phonegap technology. All platforms support Html5 so that we can make web application as a mobile one. AJAX is used to connect client and server for storing database emergency reports using MYSQL server on server side along with server scripting PHP. The haversine equation is used to compute the distance between all nearest search places from user current geolocation by using geo-coded address form emergency database search current address and provide all services under required distance. The system is designed in following integrated steps:

\subsubsection{Smartphone User Interface}

Emergency reporting application consists of cross-platform phonegap app which can run in all major operating system such as Android, iPhone, Windows. App consist of providing emergency reporting facility such as medical, fire, police emergency also to search nearest any places from the user current location such as any shop, mall etc on requirement or user can used as emergency purpose also. If user wants to report emergency to its family, friends or onto social media's such as facebook, whatsapp, twitter etc, along with location and pre-defined emergency message. If user selects emergency location as message then we provide pre-defined emergency message with his geo-location message and select contact number from mobile directory to report.

\subsubsection{Configuration of map on device using Google web services}

To use nearest location Google facility on to the Smartphone, we register onto the Google console, which provide different web services such as for used Google maps on to the mobile we need to generate server-key from console and used onto the device. To display maps onto the device we need to use Google maps followed by key and type of emergency user wants too used.

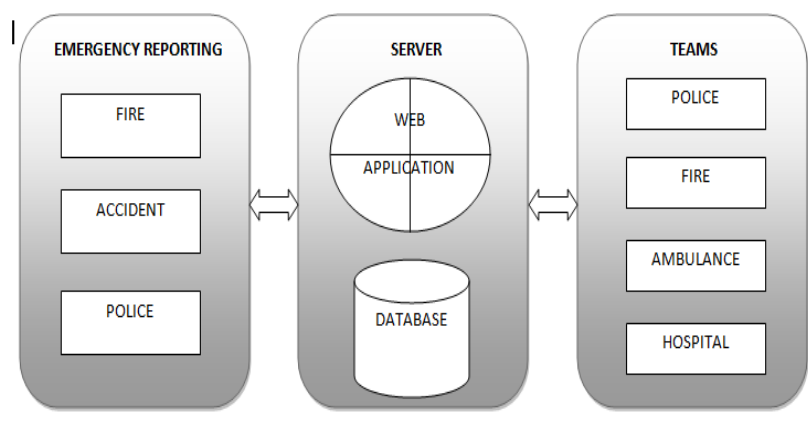

Fig1. System Architecture

\subsubsection{Build Web Server}

The PHP and MYSQL are used to design the web application server which is used to display all the emergency reports are registered and provide the nearest services from the person in an emergency. Web mapping Facility (WMS) is used to point all nearest places from the Google maps web services and GIS location based services are for displaying addresses from client position onto the maps.

\subsubsection{Build Client-Server Intermediate}

JSON (JavaScript Object notation) is a insubstantial data exchange format along with AJAX request helps to make web service connection in between client and server [17].

After the requirement is completed, the response team have been visited in place of emergency to rescue person with different facility by using emergency reporting App with server team effort.

\subsection{Technologies Used}

In this paper we used following technologies for the development of emergency reporting client-server application with all the technologies works in their original form. it is important to remember that some technologies are present only in the client (Phonegap, SQLite,JQueryMobile), some only in the server (PHP,MYSQL) and some are found on both (JSON).

\subsubsection{Phonegap}

The idea for the client was similar to the Java motto, "write once, run anywhere", pursuing to overcome the handicaps of different programming languages and very heterogeneous systems for today's mobile devices [12].

PhoneGap is a software evolution structure by Adobe System, which is utilize to develop Smartphone applications. To utilize apps using PhoneGap, the developer does not require having knowledge of mobile programming language like for android we need knowledge of Java Programming, for IOS app development objective $\mathrm{C}++$ knowledge is necessary, but only web-development and design languages like, CSS, HTML and JavaScript. PhoneGap utilizes and develope apps for all popular mobile OS platforms such as iOS, BlackBerry, Android and Windows Mobile OS etc.

With the help of apache cordova (Adobe software, previous phonegap) in visual studio 2015 Enterprise we develop crossplatform application for emergency reporting.

\subsubsection{JQueryMobile}

JavascriptQueryMobile [18] is JavaScript libraries for developing mobile application and it is also a user interface structure based on JavaScript Query that works across all popular phones, desktop platforms. Based on services and universal entrance in mind, it follows responsive web outline 
and HTML5 markup-design configuration makes to learn easy using JQueryMobile.

\subsubsection{HTML5SQL}

Transactions have been done using the html5sql library [19]: a module coded in JavaScript, implementing the Web SQL [13] A PI that eases the work with HTML5 Web Databases. The API is supported by the major web browsers (Chrome, Firefox, Opera, Safari...) including those available on the smart phones.

To create local storage of dataset we used HTML5SQL Technologies, in this paper for saving nearest location search places, favorites places are stored locally, also if user wants to add information before emergency situation occur, he can also add his details and saved locally, when emergency occurs, he can directly send information which is saved already on local database, in this paper information at the time of emergency consist of name of the user, contact number.

\subsubsection{AJAX Technology with JSON}

AJAX [20] is acronym of Asynchronous JavaScript and XML. AJAX is a new skill for creating better, efficient, faster, and more user friendly web applications with the help of Extended Markup Language, HTML, Cascading Style shit, and Java Script. this is the web browser technology autonomic of software. Any data that is updated using Asynchronous JavaScript and XML can be stored using the JavaScript Object Notation format on the web server. AJAX is necessary and used so that JavaScript can fetch these JSON files when necessary, parse them, and execute one of the following operations:

- Accumulate the parsed utilities in the variables for further procedure before displaying them on the website page.

- It immediately allocates the data to the Document Object Model elements in the website page, so that they are mention on to the website.

Example: A Nearby Search request is a Hypertext Transfer Protocol Uniform Resource Allocator of the following form:

https://maps.googleapis.com/maps/api/place/nearbysearch/out put?parameters

Where output may be either of the following values:

- JSON specify the execution in JavaScript Object Notation (JSON)

- XML specify the execution as Extended Markup Language.

Parameter consists of latitude, longitude, API-key (serverkey) etc.

\subsubsection{GIS Location based services}

Location Based Services hold a number of elements including Google maps and Geographic Information System (GIS) information, geo-location collection services, and LBS application-particular subcomponents. Location Based Service (LBS) is a good platform that facilitates information services based on the current or a known geo-location, supported by the electronic map platform or framework. The geo-location information (latitude and longitude coordinates) of Smartphone end user can be acquire through the mobile communication network or the Global Navigation Satellite Systems (GNSS) [13]. Location Based Services (LBSs) facilitate customized services to the holding-customers based on their current geo-position using Global Navigation Satellite System (GNSS), Geographical Information System (GIS) [22], Wireless Communication (WC) technology framework.

Location-based service (LBS) offers customers with data customized by the user's current geo-location, such as the nearest restaurants/hotels/hospitals, which are fetched from a spatial database stored remotely in the Location Based Services server. Location Based Services not only perform for individual Smartphone end-users, but also act as an key role in public safety, transportation facility, emergency response unit, and disaster management services. With an increasing amount of mobile devices featuring built-in Global Positioning System (GPS) technology, LBS have experienced rapid growth in the past few years onto the Smartphone users [12].

\subsubsection{Google Places API Web services}

The Google Places Application Programming Interface Web Service is a service that returns information about places defined within this Application Programming Interface as establishments, geographic locations, or prominent points of interest - using Hypertext Transfer Protocol requests.

The following place requests are available:

- $\quad$ Place Searches: it returns list of places from user's current location after placed strings in user query.

- Place Details: it returns more detailed information about a specific Place with reviews of users.

- Place Add: allows users to supplement the contents in Google's Places database with data from the user's application.

- Place Photos: It gives users to access to the millions of geo-location related photos stored in Google's Place database.

- Place Auto complete: it is used automatically to fill in the name and address of a place as you type.

- Query Auto complete: It is used to provide a query prediction service for wording-based geographic searches, by returning recommended queries as you type.

Each service is accessed as an HTTP request, and returns either a XML or JavaScript Object Notations response [16]. All requests to a Places service have to use the https:// protocol, and add an Application Programming Interface key. The Google Places Application Programming Interface Web Service uses a place ID to uniquely associate a place. For details about the appearance and usage of this recognize across the Google Places API Web Service and other API.

\subsubsection{Distance calculation}

In our paper we used the 'Haversine' formula for distance measurement for spherical measurements. It is used to compute the great-circle between pin-points-that is, the shortest distance over the spherical earth's surface [14]

Haversine formula:

$\mathrm{a}=\sin ^{2}(\Delta \varphi / 2)+\cos \varphi 1 \cdot \cos \varphi 2 \cdot \sin ^{2}(\Delta \lambda / 2)$

$\mathrm{c}=2 \cdot \operatorname{atan} 2(\sqrt{ } \mathrm{a}, \sqrt{ }(1-\mathrm{a})) \mathrm{d}=\mathrm{R} \cdot \mathrm{c}$

Where 
$\Phi$ is latitude, $R$ is earth's radius, (mean radius $=6371000$ meters), $\lambda$ is longitude; the angles should be in radians to pass to trig functions!

$$
\begin{aligned}
& \operatorname{var} \mathrm{R}=6371 ; / / \mathrm{km} \\
& \operatorname{var} \varphi 1=\text { lat1.toRadians(); } \\
& \operatorname{var} \varphi 2=\text { lat2.toRadians(); } \\
& \operatorname{var} \Delta \varphi=(\text { lat2-lat1).toRadians(); } \\
& \operatorname{var} \Delta \lambda=(\operatorname{lon} 2-\text { lon1).toRadians(); }
\end{aligned}
$$

In this paper, we calculate shortest distance between user address location and nearest places in $\mathrm{Km}$ using Google web mapping facility to map these places and name of the places displayed onto it with shortest distance.

\section{EXPERIMENTAL WORK}

\subsection{Client side Graphical user Development}

We design GUI for emergency reporting to the person in emergency with exact geo-location (latitude and longitude) using GIS Location based service technology. The main Emergency application shown in fig.3 (a)-(h), the step by step instruction are given in "how to use App" section, but here we discuss how actually it works, starting from the fig.3 (a), consist of main start-up page of the App consist of 6 menu options from which we given 3 options for the emergency service providers, one is for providing by own emergency and one is to find place of interest nearby for the user. We provided medical, fire, police emergency services for the rescue person. To get emergency services person have to select which type of available emergency he/she wants to uses. let's consider person at emergency selected emergency services, from fig.3 (b) shows the information have to filled by the person at emergency to get the list of nearby hospitals from incident location (person's present location), once the correct information filled, data will be saved to MYSQL server from fig.3 (c) and within quick time emergency will be provided to the person, there will be two option for the person in emergency to get emergency service either get service their own or from server data filled team, from the fig.3 (d) list of nearest hospitals will be displayed, per request from the current location 20 nearest hospitals or emergency services will displayed. In urban areas if person is localities he will be aware of the hospitals nearest to him/her, but don't have contact information to be saved on mobile, so this will get him to get information with contact numbers and the hospital that he/she wants. This will also helpful for the person new to the city and in emergency and but don't know about nearest or good hospitals, so from fig.3 (e), he/she can also get and help to get service with information. Contact numbers are provided here so that can get own services by calling their own or someone near to him/her and get service, this information system will available not only for the Aurangabad city as a case study, this app will use everywhere to get services. Now, form fig.3 (f) user can also get mapping facility to see where he is exactly and the nearest hospitals/fire stations/police stations from his locations, all the nearest service places mapped with the using Google map web services onto the app. In this way someone new to the city can find and get the nearest facility and get its own or with server response to the request. These all are the emergency reporting facility from the app which we provided, also there is an option for the person in an emergency to get help from the family members or any group from the social media's in case of emergency. This function will selects number of contact numbers from the mobile phone and share them via sms your current location and compose pre-defined emergency message from fig. 3 (h) so that they will help. Also can share location onto social media such facebook, whatsapp etc. To share locations with emergency message as shown in fig. $3(\mathrm{~g})$.

\subsection{Server side Development}

Server-side of the system consist of reported emergency listbox where all the complaint reported are saved to MYSQL Database and it will update with request, server have responsibility to keep update of new request arrival. All the reported emergency save's with information like type of the emergency complaint, date with time, geo-coded address of the place, latitude and longitude (spatial data), once the emergency arrival server have responsibility to check from where the emergency comes from, by using geo-coded address (road network address) server will find the exact areas from where emergency comes, also from the spatial data server can also find out the precise location of the emergency comes where from. Once the area server get then has to select the required emergency such as medical for hospitals with ambulance, fire station facility or police facility, server have option within meters search the facility such as 500 meters, 1000 meters, 5000 meters etc. And when complete with all search click on search to display the name of the emergency type along with shortest distance, which we calculated using haversine formula, which gives server precise location with shortest distance from the person in an emergency as shown in fig.2 the purpose behind save data to server from mobile application is to make analysis from the collected database in future when application get's old and large amount of data will available and we will make predictions on data gathered, how to prevent all these emergencies, makes sets of rules, can analyzes in which area emergency reports makes high etc. 


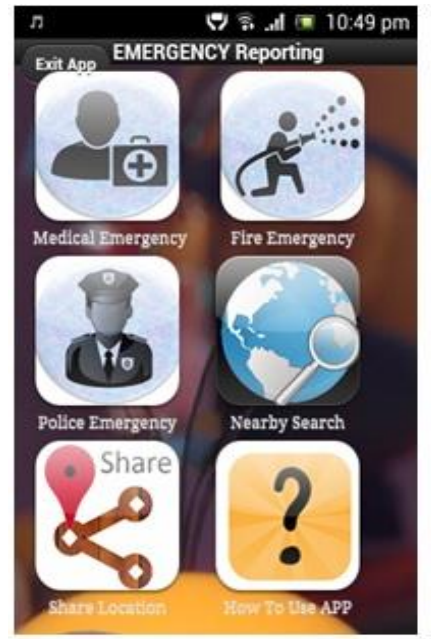

(a)

\begin{tabular}{|c|c|}
\hline \multirow{2}{*}{$\begin{array}{l}\text { Back } \\
\text { Back }\end{array}$} & (1) F at a $10: 50 \mathrm{pm}$ \\
\hline & Hospital Details \\
\hline \multicolumn{2}{|r|}{ Search Details } \\
\hline Name & Dr Hedgewar Hospital \\
\hline Address & $\begin{array}{l}\text { Trimurthi Chowk,Gajanand } \\
\text { maharaj Mandir, Garkheda, } \\
\text { Aurangabad, Maharashtra } \\
\text { 431005, India }\end{array}$ \\
\hline Phone & 02402339866 \\
\hline Rating & 4.4 \\
\hline & Add to Favorite \\
\hline & Visit HomePage \\
\hline
\end{tabular}

(e)

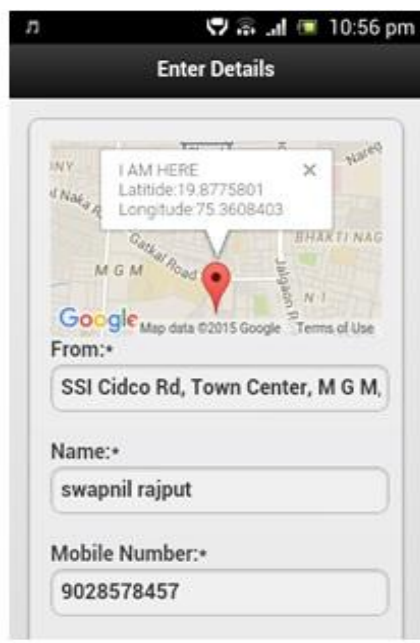

(b)

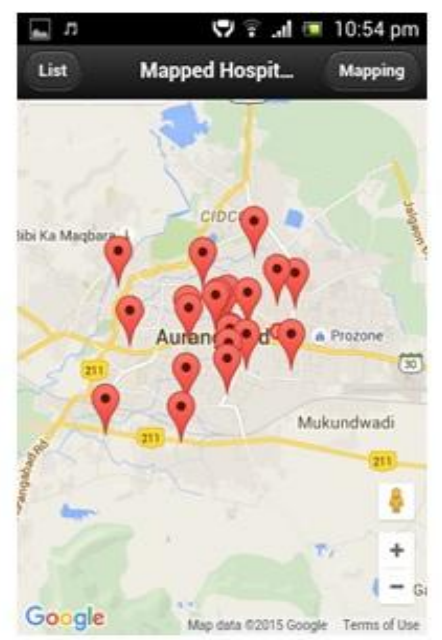

(F)

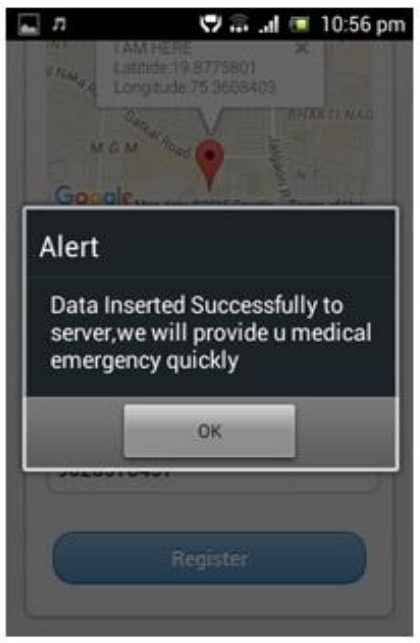

(c)

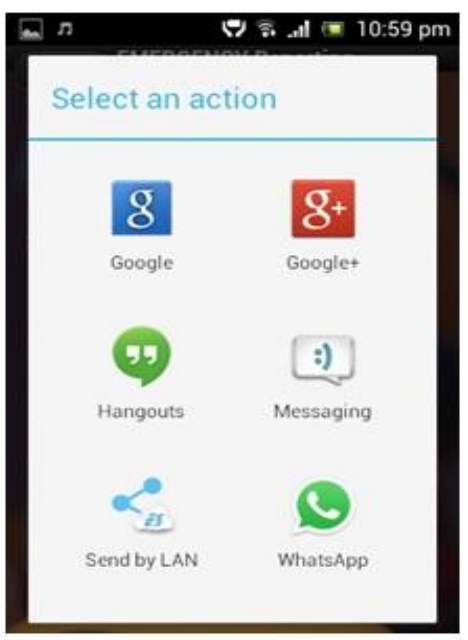

(g)

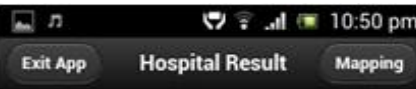

凹 Sakolkar Charitable Hospit. vicinity:Bhakti Nagar, N1, Cidco, Au. (3) Index:0

G Bembde Hospital vicinity:Beed Bypass Road, Auranga. () Index:1

(1) Dr Hedgewar Hospital vicinity:Trimurthi Chowk, Gajanand Index:2

(1) Govt. Dental Hospital \& Co. vicinity:Navkhanda, Aurangabad Index:3

(d)

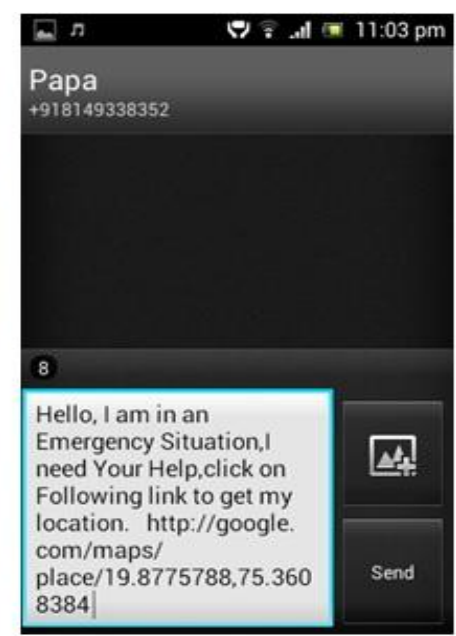

(h)

Fig 2. GUI of Smartphone Emergency APP

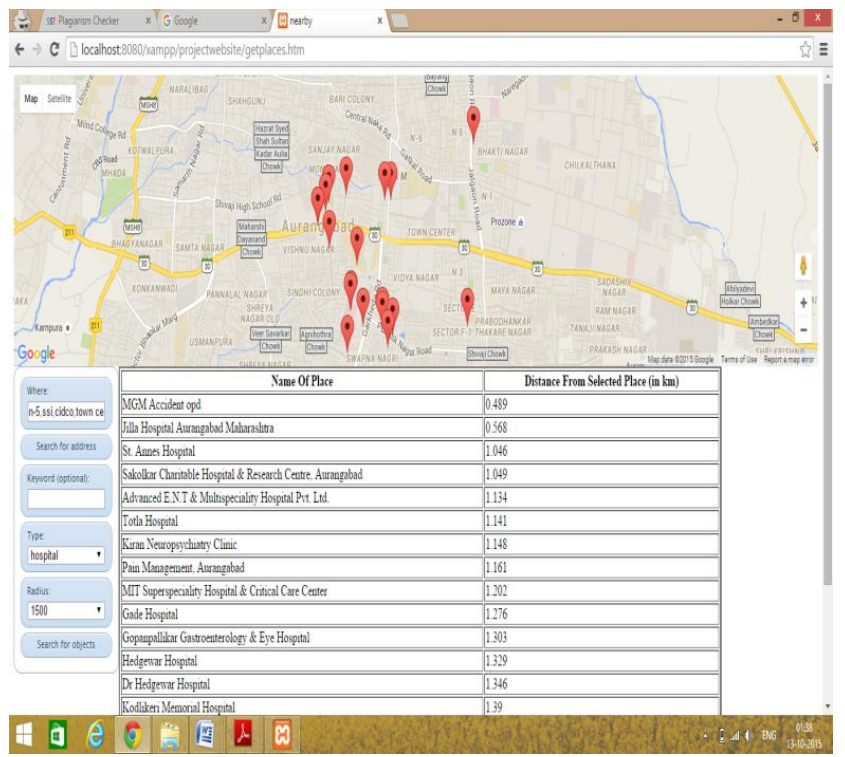

Fig 3. Web-server location search with shortest distance

\section{CONCLUSIONS}

This paper gives an idea about new emergency reporting system using GIS Location based and Google web services for in the any emergency using Smartphone's by providing the help by rescue team. It responds to citizen emergencies in urban areas and Emergency management fulfils the requirement of ad-hoc communication between skilled personnel for emergency times when there is no other communication media exists.

Emergency never comes with prior information, using this location and GIS integrated mapping system we can automatically detect precise location (latitude and longitude) of the person in emergency and provide services from Emergency management team.

In this paper, we add another option for communication using mobile and web technologies for emergency reporting and response. This will help for fast and efficient emergency units to dispatch. Our goal is not just create emergency reporting and response protocol, in modern era we have just maximize the use of Smartphone's which act as a medium and to help peoples, save their lives in case of emergency. This will also help for command centre/emergency unit to easily plot the 
location of on a map. In future, we can make more precise client-server application to help peoples by analyzing from the previous database records and make predications on how emergency cases occurs, in which urban areas emergency occurs most of the times. Even further we can make the provision of sending the pictures of emergency situations with geo-tagged images.

\section{ACKNOWLEDGMENT}

The authors would like to acknowledge and thanks to University Grants Commission (UGC), India for granting UGC SAP (II) DRS Phase-I \& Phase-II F. No. 3-42/2009 \& 4-15/2015/DRS-II for Laboratory facility to Department of Computer Science and Information Technology, Dr. Babasaheb Ambedkar Marathwada University, Aurangabad, Maharashtra, India and financial assistance under UGC -BSR Fellowship for this work.

\section{REFERENCES}

[1] Fuming Shih, Oshani Seneviratne, Ilaria Liccardi, Evan Patton, Patrick Meier, Carlos Castillo, "Democratizing mobile app development for disaster management ", AIIP '13 Joint Proceedings of the Workshop on AI Problems and Approaches for Intelligent Environments and Workshop on Semantic Cities, Pages 39-42.

[2] Appdynamics,"https://www.appdynamics.com/media/upl oadedfiles/White_Paper_Going_live_with_a_mobile_app _1.pdf" [Accessed: Oct 15, 2015].

[3] DeeleshMandloi\& Rajiv Gupta,|Evaluation of accident black spots on roads using Geographical InformationSystems (GIS)\|, Map INDIA Conference, 2003

[4] Apparao. G, P. Mallikarjunareddy Dr. SSSV Gopala Raju,IIdentification Of Accident Black Spots For National Highway Using GIS\|, INTERNATIONAL JOURNAL OF SCIENTIFIC \& TECHNOLOGY RESEARCH, VOLUME 2, ISSUE 2,2011.

[5] Telecom Regulatory Authority of India: trai.gov.in/.../Consultation\%20paper\%20IECS\%2015-313.pdf.

[6] Aurangabad Municipal Corporation (AMC): http://www.aurangabadmahapalika.org/complaint.jsp?id $=14$ [Accessed: May. 17, 2015].

[7] Swapnil R.Rajput and K.V.Kale, "A Review Paper on GIS Web-System to Support Emergency Situations in Urban Areas and Provide Services", International Journal of Computer Sciences and Engineering, Volume-03, Issue-05, Page No (345-350), May -2015, E-ISSN: 23472693.

[8] Official ELERTS Website. (2013, January). [Online]. Available: http://elerts.com

[9] Osnat Mokryn, Dror Karmi, Akiva Elkayam, Tomer Teller "Help Me: Opportunistic Smart Rescue
Application and System" The 11th Annual Mediterranean Ad Hoc Networking Workshop (MedHoc-Net), 2012.

[10] Komwit Surachat, Supasit Kajkamhaeng, Kasikrit Damkliang, Watanyoo Tiprat, and aninnuch Wacharanimit, "First Aid Application on Mobile Device”, International Scholarly and Scientific Research \& Innovation 7(5) 2013,pp-361-366.

[11] Official GreatCall Website. (2013, January). [Online]. Available: http://www.greatcall.com

[12] Kumar, S.; Qadeer, M.A.; Gupta, A., "Location based services using android (LBSOID)," in Internet Multimedia Services Architecture and Applications (IMSAA), 2009 IEEE International Conference on, vol., no., pp.1-5, 9-11 Dec. 2009.

[13] Q Ren, M. H. Dunham, Using Semantic Caching to Manage Location Dependent Data in Mobile Computing, In the $6^{\text {th }}$ Annual International Conference on Mobile Computing and Networking, Boston: ACM Press, 2000, pp.210-222.

[14] Harversine Algorithm viewd on: http://www.movabletype.co.uk. [Accessed: September. 17, 2015].

[15] Google places API Web Services "https://developers.google.com/places/web-service/getapi-key" [Accessed: September. 17, 2015].

[16] Gaikwad, D.B.; Wanjari, Y.W.; Kale, K.V., "Disaster management by integration of web services with geospatial data mining," India Conference (INDICON), 2014 Annual IEEE, vol., no., pp.1-6, 11-13 Dec. 2014.

[17] Jethro B. de Guzman, Ritz Carlo C. de Guzman, and Engr. Remedios G. Ado," Mobile Emergency Response Application Using Geolocation for Command Centers", International Journal of Computer and Communication Engineering, Vol. 3, No. 4, 2014,pp.235-238.

[18] JQuery Mobile "https://jquerymobile.com/" [Accessed:August 16,2015]

[19] SQLite Database "https://www.sqlite.org/" [Accessed: August 16,2015]

[20] AJAX Technologies http://www.tutorialspoint.com/ajax/ ajax_technology.htm [Accessed:August 16,2015]

[21] Calculate distance, bearing and more between Latitude/Longitude points. http://www.movabletype.co.uk/scripts/latlong.html. [Accessed: sept 17,2015]

[22] Li Zhigang; Liangtian; Yang Wunian, "Research of GISbased urban disaster emergency management information system," Computer and Communication Technologies in Agriculture Engineering (CCTAE), 2010 International Conference On , vol.2, no., pp.484487, 12-13 June 2010. 